\title{
Requisitos de usabilidade para softwares aplicados ao e-learning: uma proposta para elaboração de User Stories
}

\author{
Larissa A. Lopes $^{1}$, Eduardo G. Pinheiro ${ }^{1}$, Tiago S. da Silva ${ }^{2}$, Luciana A. M. Zaina ${ }^{1}$ \\ ${ }^{1}$ UFSCar - Universidade Federal de São Carlos \\ Sorocaba - SP - Brasil \\ ${ }^{2}$ UNIFESP - Universidade Federal de São Paulo \\ São José dos Campos - SP - Brasil \\ \{larii.albano, edu.g.pinheiro\}@gmail.com, \\ silvadasilva@unifesp.br, lzaina@ufscar.br
}

\begin{abstract}
Software development for e-learning has become increasingly complex, just as User Stories (US) are artifacts that have been widely used in the conception phase of the software. This paper presents an approach to support developers in the writing of US that addresses usability requirements in the elearning domain. An experimental study was conducted with 19 participants that evaluated positively the use of the approach. The results also revealed that the usability guidelines for e-learning are an important tool for specifying usability aspects into the US.
\end{abstract}

Resumo. O desenvolvimento de software para e-learning vem se tornando cada vez mais complexo, assim como User Stories (US) são artefatos que tem sido artefatos amplamente utilizados na fase de concepção do software. Este artigo apresenta uma abordagem para apoiar os desenvolvedores na escrita de US que contemplem requisitos de usabilidade no domínio de e-learning. Foi realizado um estudo experimental com 19 participantes que avaliaram positivamente o uso da abordagem. Os resultados revelaram que as diretrizes de usabilidade para e-learning auxiliaram os participantes na especificação de aspectos de usabilidade.

\section{Introdução}

A complexidade é inerente ao desenvolvimento de software para aprendizagem eletrônica (e-learning). Esta complexidade é impactada pela diversidade de funcionalidades, necessidade de interoperabilidade de infraestrutura de terceiros (por exemplo, repositórios de materiais de aprendizagem, redes sociais) e participação de diferentes stakeholders (partes interessadas), como desenvolvedores, designers, alunos, professores, pedagogos, sistemas regulatórios governamentais, entre outros [Chimalakonda and Nori 2012]. Contudo, o desenvolvimento destes softwares é muitas vezes carente no uso de técnicas e métodos de Engenharia de Software (ES) [Dodero et al. 2014, Chimalakonda and Nori 2012]. Além disto, muitas vezes as técnicas e métodos de ES precisam ser adaptadas de forma a convergir para particularidades importantes deste domínio [Sarasa-Cabezuelo and Sierra-Rodríguez 2013]. 
VIII Congresso Brasileiro de Informática na Educação (CBIE 2019)

Anais do XXX Simpósio Brasileiro de Informática na Educação (SBIE 2019)

As abordagens de desenvolvimento ágil de software (DAS) têm sido amplamente adotadas para diferentes domínios, incluindo e-learning [Dodero et al. 2014]. Frequentemente, as funcionalidades e características de qualidade do software são descritos através de User Stories (US) [Schön et al. 2017], um artefato popularmente usado pelos desenvolvedores de software que adotam o DAS. Dada a importância do uso das USs, muitos trabalhos têm proposto formas diferenciadas de incluir aspectos de qualidade, como a usabilidade, dentro da descrição de US [Hudson 2013, Choma et al. 2016].

A usabilidade é um dos aspectos de qualidade que deve ser considerado desde a concepção de um software [Van Nuland et al. 2017]. A ISO [9241-210 2009] define usabilidade como uma medida que especifica o quanto um produto pode ser utilizado por certos usuários para atingir funcionalidades determinadas. Dado seu contexto de uso, esta funcionalidade deve ser atingida com eficácia, eficiência e satisfação. Lidar com a usabilidade de um software para e-learning pode trazer benefícios para a melhoria da prática educacional [Gonçalves et al. 2014], impactando na boa experiência do usuário. Isto evita que o usuário desvie o foco das tarefas de aprendizagem para aprender a utilizar corretamente o software [Gordillo et al. 2014].

A usabilidade relativa a um domínio pode ser considerada a partir do conhecimento sobre as necessidades dos usuários finais. Para isto, a área de Interação HumanoComputador (IHC) provê um conjunto de técnicas, métodos e artefatos que auxiliam o desenvolvedor no entendimento de diversos aspectos sobre o usuário [Rogers et al. 2013]. Outra forma de considerar a usabilidade é utilizando diretrizes de usabilidade [Nakamura et al. 2017]. Embora existam diversas diretrizes de usabilidade genéricas, estas não contemplam características específicas, demandando um esforço maior da atenção do desenvolvedor sobre particularidades de um dado domínio [Quiñones and Rusu 2017]. Diversas diretrizes de usabilidade para o domínio de e-learning foram propostas nos últimos anos [Van Nuland et al. 2017, Nakamura et al. 2017].

A comunidade Brasileira de Informática na Educação tem apresentado iniciativas relacionadas à Engenharia de Software para e-learning. Temas já abordados são: reúso de software [Dalmon and Brandão 2013, Zaina et al. 2015], processos de software [Brito 2013], elicitação de requisitos [Silva et al. 2018] e integração do design de interação ao processo de ES [Santos et al. 2017, Ribeiro and Melo 2017]. Contudo, observa-se a pouca articulação sobre o uso das diretrizes de usabilidade juntamente com as técnicas e métodos de ES. Também não foram encontradas até o momento propostas que dessem suporte à escrita de US considerando aspectos de usabilidade focados ao ambiente de e-learning.

Este artigo tem por objetivo apresentar a UsaUSe (Usabilidade em User Stories para e-learning), uma abordagem para apoiar desenvolvedores de software na escrita de US para o domínio de e-learning. A proposta é baseada na triangulação do uso de uma gramática específica para escrita de US em conjunto com a adoção de artefatos de IHC e diretrizes de usabilidade para e-learning. A proposta foi avaliada por 19 participantes com experiência em DAS e IHC. A principal contribuição deste trabalho é prover uma abordagem que foca em aspectos relevantes ao desenvolvimento de software para $e$-learning desde a sua concepção, utilizando para isto um artefato amplamente usado no DAS - User Stories. 
VIII Congresso Brasileiro de Informática na Educação (CBIE 2019)

Anais do XXX Simpósio Brasileiro de Informática na Educação (SBIE 2019)

\section{Fundamentação Teórica}

A fase de concepção de um software é considerada crítica e fundamental para que as demais etapas de desenvolvimento possam ser realizadas adequadamente [Gordillo et al. 2014]. O entendimento sobre o usuário é considerado o ponto de partida para determinar funcionalidades ou aspectos de qualidade do software. Os desenvolvedores que atuam no DAS usualmente formalizam este entendimento escrevendo as USs. A US é um artefato importante para o time desenvolvedor, pois permite melhorar o entendimento e a comunicação em relação ao que deve ser implementado no sistema [Schön et al. 2017].

[Cohn 2009] propõe uma estrutura, que hoje é a mais utilizada dentro do DAS, na forma de gramática para descrição de uma US: "Como um <tipo de usuário $>$, Eu quero <algum objetivo > para que [alguma razão]". Sendo os dois primeiros elementos obrigatórios e a razão opcional. Junto à US são descritos os Critérios de Aceitação (Acceptance Criteria - AC). Os AC são utilizados para apontar trechos críticos no desenvolvimento da US que auxiliam a equipe tanto na fase do desenvolvimento quanto na fase de testes.

Incorporar requisitos de usabilidade desde a concepção do software potencializa a aceitação dele pelos usuários finais [Gordillo et al. 2014]. Existe uma ampla variedade de técnicas e métodos de IHC que provêm artefatos com informações sobre o usuário final que são coletadas por especialistas em IHC [Rogers et al. 2013]. Diretrizes de usabilidade - guias com critérios e atributos de usabilidade utilizados para orientar desenvolvedores e stakeholders na construção e na avaliação de uma aplicação - podem ser um destes artefatos.

\section{Trabalhos relacionados}

Embora a proposta de [Cohn 2009] seja a mais usada, alguns trabalhos surgiram com o objetivo de inserir requisitos de usabilidade na descrição das US. [Choma et al. 2016] apresentam a UserX Story, uma gramática cujo elementos são relacionados a três diferentes artefatos de IHC: as 10 heurísticas de Nielsen (diretrizes genéricas de usabilidade), Personas (descrição de personagens fictícios que descrevem um grupo de usuários) e cenários de interação (descrevem a interação do usuário com o software) [Rogers et al. 2013]. [Hudson 2013] sugere uma gramática adotando a técnica de Persona como parte da descrição da US. [Moreno and Yagüe 2012] propõem a incorporação dos requisitos de usabilidade através da adição de novas US ou modificação de tarefas existentes nas US. Já [Silva et al. 2018] propõem a adaptação de um artefato inspirado em Personas, juntamente com um questionário guia para especificação de jogos educacionais. Esses trabalhos são importantes para a área, porém nenhum deles aborda tal construção em aplicações para e-learning. Acredita-se que o domínio de aplicações para e-learning seja repleto de especificidades importantes que devam ser consideradas nas US. Além disto, apenas [Choma et al. 2016] e [Moreno and Yagüe 2012] buscam incorporar questões de UX e usabilidade nas US, mas ambos os trabalhos não apresentam validações que pudessem extrair a contribuição da proposta em um domínio específico.

Nos últimos anos, diretrizes de usabilidade para domínios específicos vêm sendo desenvolvidas como uma maneira de complementar as diretrizes genéricas [Quiñones and Rusu 2017, Nakamura et al. 2017]. Para avaliar uma aplicação para $e$ - 
VIII Congresso Brasileiro de Informática na Educação (CBIE 2019)

Anais do XXX Simpósio Brasileiro de Informática na Educação (SBIE 2019)

learning é necessário preocupar-se com requisitos de usabilidade tanto de uso do software como pedagógica. [Ardito et al. 2006] propõem um conjunto de 39 diretrizes de usabilidade para o domínio de $e$-learning. Essas diretrizes são agrupadas em quatro dimensões que são: (i) apresentação - contempla elementos vinculados ao design visual das ferramentas; (ii) hipermídia - foca em aspectos relacionados a personalização e navegação dentro de um ambiente de e-learning; (iii) proatividade da aplicação - visa prover recursos que apoiem as atividades dos alunos; e (iv) atividade do usuário - dar suporte às necessidades do estudante e professor com foco no ensino-aprendizagem. Dentro de cada dimensão, as diretrizes são divididas a partir dos princípios de eficácia e eficiência. A definição de eficácia e eficiência são adaptadas para as especificidades do domínio de e-learning. As diretrizes de [Ardito et al. 2006] têm sido usadas como referência por diversos trabalhos na área de e-learning [Van Nuland et al. 2017, Nakamura et al. 2017].

\section{UsaUSe: Usabilidade em User Stories para e-learning}

A Figura 1 apresenta a UsaUSe que é composta por três elementos. As diretrizes de usabilidade propostas por [Ardito et al. 2006] dão suporte para que o desenvolvedor tenha em mente aspectos fundamentais dentro do domínio de e-learning. O conjunto de artefatos de IHC reportam especificidades dos usuários dentro do contexto do software que se deseja construir. A gramática direciona o desenvolvedor sobre quais aspectos são importantes de serem descritos. A gramática proposta foi concebida a partir dos trabalhos relacionados a escrita de USs (ver Seção 3) e é descrita a seguir.

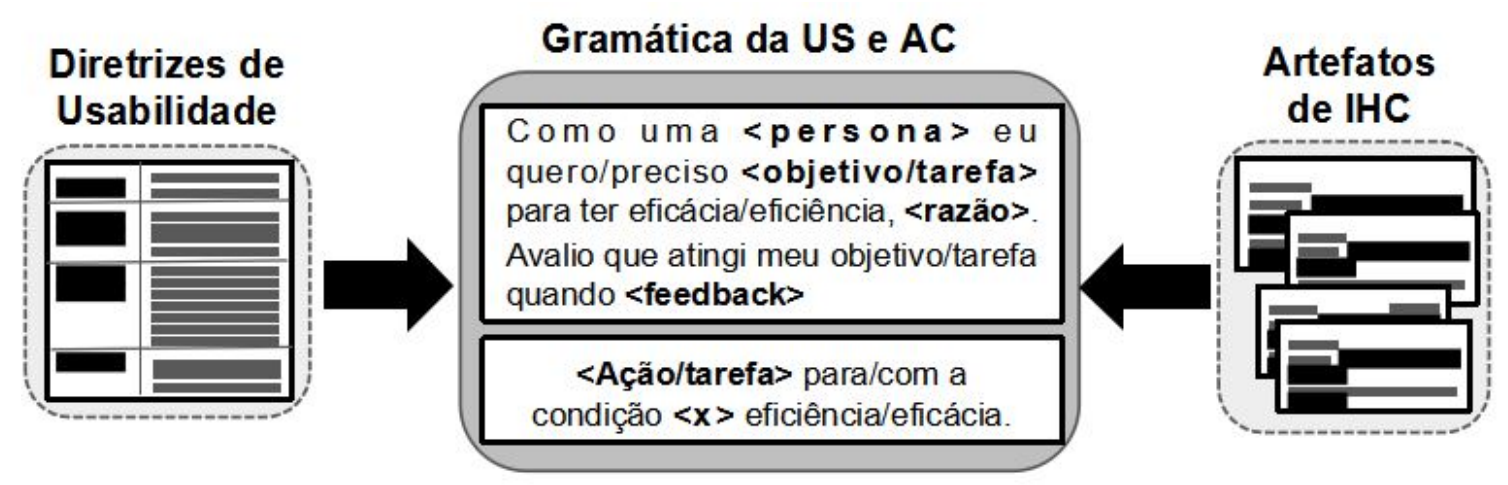

Figura 1. Abordagem UsaUSe - elementos variáveis encontram-se entre $<>$

Os elementos $<$ objetivo $>$ e $<$ razão $>$, existentes na proposta de [Cohn 2009], permanecem na UsaUSe. Estes elementos foram mantidos para informar de maneira objetiva o foco da US [Cohn 2009]. O elemento $<$ Persona $>$ substituiu o elemento $<$ tipo de usuário $>$. Esta abordagem já havia sido usada pelos trabalhos de [Choma et al. 2016] e [Hudson 2013]. A técnica de Persona estimula nos desenvolvedores o sentimento de empatia pelos usuários, pois reporta os objetivos, necessidades e dificuldades daquele determinado grupo de usuários [Rogers et al. 2013].

O elemento $<$ Feedback $>$, usado por [Choma et al. 2016], foi incorporado para que os desenvolvedores estejam atentos à importância de manter o usuário informado durante a interação com o software. Isto traz um impacto importante e relevante para a área de e-learning, pois mantém o aluno ou professor informado de sua interação. Considerando as propostas de [Choma et al. 2016] e [Moreno and Yagüe 2012], foram sugeridos 
VIII Congresso Brasileiro de Informática na Educação (CBIE 2019)

Anais do XXX Simpósio Brasileiro de Informática na Educação (SBIE 2019)

dois elementos para escrita dos ACs. O intuito do AC é testar a US através de uma $<a c ̧ \tilde{a} o\rangle$. No elemento $\langle x\rangle$ deve ser informado qual a condição que deve ser atendida para que a $<a c ̧ \tilde{a} o>$ atenda a US descrita. Embora estes elementos tenham sido inspirados pela proposta de [Choma et al. 2016] e [Moreno and Yagüe 2012], a UsaUSe diferenciase por ser mais flexível e não atrelar os elementos a nenhum artefato de IHC específico.

\section{Avaliando o uso da UsaUSe}

Um estudo experimental foi conduzido para a avaliação da UsaUSe. O objetivo principal foi verificar a aceitação da abordagem na visão dos desenvolvedores e quais diretrizes e artefatos de IHC eles utilizariam e considerariam relevantes para a concepção da USs. Escolheu-se o tema Museu Virtual de Aprendizagem, pois o grupo de pesquisa já desenvolvera um trabalho prévio sobre este tema e possuía um conjunto de artefatos de IHC maduros que reportavam informações sobre o usuário final. Os participantes deveriam especificar US para um software que permitisse a aprendizagem de crianças e adolescentes sobre os temas de História e Artes a partir de museus virtuais, incluindo acesso por meio de dispositivos móveis.

Considerando o objetivo, duas perguntas foram delineadas: (P1) Quais foram as diretrizes de usabilidade utilizadas pelos desenvolvedores na escrita das USs?; e (P2) Quão fácil e útil os desenvolvedores consideram utilizar diretrizes de usabilidade para escrever as UsaUS?. Todos os artefatos usados no estudo experimental descritos a seguir estão acessíveis ${ }^{1}$. Participaram da avaliação 19 desenvolvedores de software, sendo que 7 faziam parte do curso de graduação em Computação da UFSCar e 12 do programa de pós-graduação em Ciência da Computação do INPE. Os estudantes de graduação encontravam-se em estágio avançado do curso, já realizando estágio em empresas. Um questionário de caracterização coletou o perfil e o conhecimento dos participantes sobre o domínio estudado e às técnicas/métodos de IHC e ES.

O estudo foi conduzido em apenas um dia com duração de 4 horas para cada grupo (UFSCar e INPE), porém em dias diferentes nas duas universidades. Um estudante de mestrado e um professor doutor, atuantes em IHC e ES, aplicaram na UFSCar. O mesmo professor doutor replicou o estudo na INPE com auxílio de outro professor doutor, com experiência em IHC e ES. Antes de realizar a escrita das USs os participantes receberam um treinamento, de aproximadamente 2 horas, que visava nivelar os conhecimentos sobre escrita de US e desenvolvimento de software para e-learning. Para mitigar possíveis problemas de cansaço dos participantes durante o estudo, foi realizado um breve intervalo entre o treinamento e a execução do estudo experimental.

Foi disponibilizado um conjunto de artefatos de IHC e ES que continham informações sobre os usuários e o domínio. Estes já haviam sido utilizados previamente por outros desenvolvedores de software. Haviam artefatos de IHC referentes as seguintes técnicas/métodos: Cenário de interação; Persona; resultados do Card-Sorting realizado com usuários alvos; StoryBoard; 10 heurísticas de Nielsen; Questionários contendo informações sobre necessidades e hábitos dos usuários alvos; e Teste de Usabilidade contendo dados sobre a eficiência e eficácia do uso de um protótipo de média fidelidade da aplicação. No caso de ES, os artefatos eram referentes à: lista de Requisitos Funcionais e a descrição do tema. Foi elaborado um template onde os participantes escreveriam as

\footnotetext{
${ }^{1}$ http://uxleris.sor.ufscar.br/uxleris/index.php/fields-studies/
} 
VIII Congresso Brasileiro de Informática na Educação (CBIE 2019)

Anais do XXX Simpósio Brasileiro de Informática na Educação (SBIE 2019)

US (no centro do artefato) e deveriam descrever os artefatos e diretrizes utilizados através de post-its. O template já continha a gramática proposta na abordagem. Além disto, foi disponibilizado um artefato contendo as diretrizes de usabilidade de [Ardito et al. 2006].

Ao final do estudo experimental foram produzidas 33 USs. Os participantes responderam a um questionário baseado no modelo de aceitação tecnológica TAM (Technology Acceptance Model) para coletar a percepção sobre o uso das diretrizes de usabilidade no apoio à escrita das US. Esse questionário foi proposto por [Davis 1989] e adaptado para a avaliação das diretrizes.

A análise dos dados contou com a participação de dois pesquisadores mestrandos e dois doutores, todos especialistas em IHC e ES. Em uma primeira etapa, todas as 33 US e seus respectivos AC foram analisados separadamente por dois mestrandos da área de IHC e os resultados depois consolidados. Numa segunda etapa, os professores doutores refinaram os resultados gerados no primeiro ciclo, discutindo junto aos mestrandos os resultados finais. A Figura 2 apresenta um exemplo de uma US elaborada por um participante do estudo.

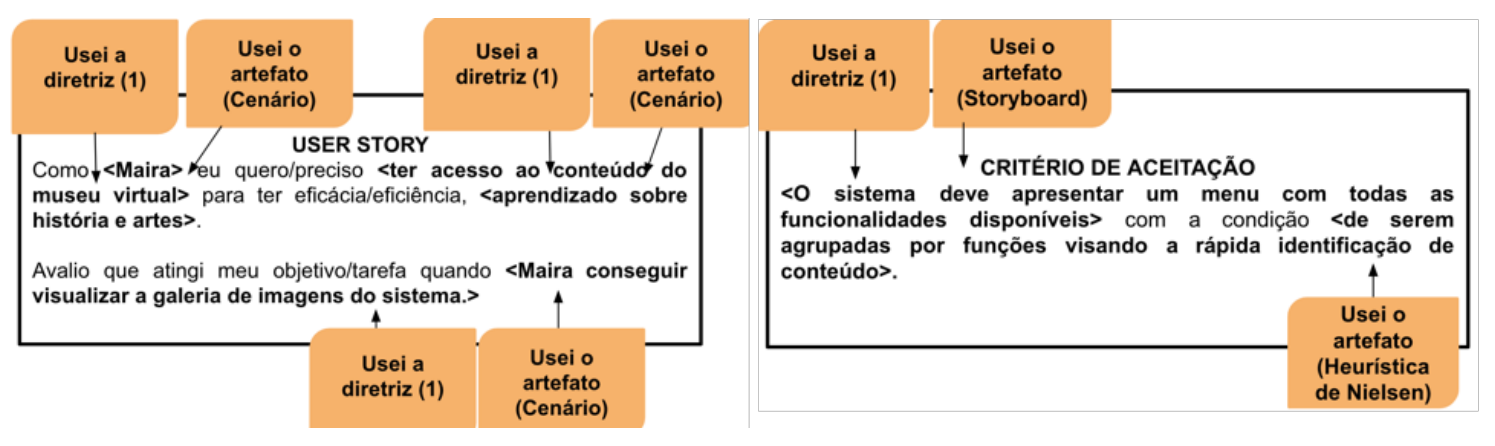

Figura 2. US elaborada por participante da UNIFESP

\section{Resultados e Discussão}

Foi possível constatar que Prototipação, Questionários, Entrevistas e Teste de usabilidade são as técnicas de IHC mais conhecidas pelos participantes. Também foi possível verificar que os participantes tinham algum conhecimento sobre US. Das 33 US elaboradas pelos 19 participantes, 12 foram escritas por alunos de doutorado, 11 por alunos de mestrado e 7 por alunos de graduação. Observando os dados sobre a experiência prática dos participantes contatou-se que os os mais experientes escreveram uma maior quantidade de US.

Para responder a (P1) Quais foram as diretrizes de usabilidade utilizadas pelos desenvolvedores na escrita das UsaUSe?, foram realizados dois tipos de investigação. Primeiro, foram verificadas quais diretrizes foram usadas em cada elemento da gramática. Durante a execução do estudo foi informado aos participantes que eles deveriam utilizar as diretrizes que achassem pertinente para auxiliá-los na escrita; e que não seria necessário utilizar todas elas. Os resultados revelaram que as dimensões "Apresentação" e "Atividade do Usuário" foram as mais utilizadas considerando todos os elementos da gramática. A diretriz mais apontada foi a ("Fornecer mecanismos de pesquisa por indexação, chave ou linguagem natural.") que está diretamente relacionado a busca de obras de arte no museu e tem relação tanto com requisito funcional (Fornecer mecanismos de pesquisa...) quanto 
VIII Congresso Brasileiro de Informática na Educação (CBIE 2019)

Anais do XXX Simpósio Brasileiro de Informática na Educação (SBIE 2019)

com diferentes formas de prover essa funcionalidade ao usuário (por indexação, chave ou linguagem natural).

Também foi possível observar que em todos os elementos da gramática as diretrizes mais apontadas pertencem a dimensão "Apresentação". Observou-se que houve um grande número de apontamentos de uso das diretrizes para a descrição de $<\mathrm{x}>$ que diz respeito as condições que devem ser atendidas pelos AC. O que representa que as diretrizes deram suporte a escrita de $\mathrm{AC}$ mais direcionados as características do domínio. Isto auxilia a equipe tanto na fase do desenvolvimento do software quanto na fase de teste. Também observou-se que em alguns casos os participantes apontaram que as próprias diretrizes de usabilidade os auxiliaram na escrita da US/AC.

\begin{tabular}{c|c|c|c|c}
\hline \hline Dimensão & Objetivo & Razão & Feedback & Ação/X \\
\hline Apresentação & C, P, S, TU & C, HN, P, LRF, S & C, DU, HN, P, LRF, S & $\begin{array}{c}\text { C, DU, HN, Q, P, } \\
\text { LRF, S, TU }\end{array}$ \\
\hline Hipermídia & CS, C, P & P, LRF, S & P, LRF & C, P, LRF, TU \\
\hline $\begin{array}{c}\text { Proatividade } \\
\text { da aplicação }\end{array}$ & C, P, Q, S, TU & C, DT, DU, P & DU, C, P, Q, S, TU & C, DT, DU, P, Q \\
\hline $\begin{array}{c}\text { Atividade } \\
\text { do usuário }\end{array}$ & $\begin{array}{c}\text { C, DU, HN, P, } \\
\text { LRF, S }\end{array}$ & $\begin{array}{c}\text { C, DU, Q, P, } \\
\text { LRF, S }\end{array}$ & DU, P, Q, S & $\begin{array}{c}\text { C, DU, HN, P, } \\
\text { Q, S, LRF, TU }\end{array}$ \\
\hline
\end{tabular}

Tabela 1. Mapeamento das dimensões das diretrizes de usabilidade $\mathrm{x}$ elementos da gramática $x$ artefatos de IHC - Card Sorting (CS), Cenário (C), Descrição do Tema (DT), Diretrizes de Usabilidade (DU), 10 Heurísticas de Nielsen (HN), Lista de Requisitos Funcionais (LRF), Persona (P), Questionário (Q), Storyboard (S), e Teste de Usabilidade (TU)

Uma segunda análise buscou mapear o uso das diretrizes de usabilidade (por dimensão), artefatos de IHC utilizados e elementos da gramática onde estes foram aplicados. A Tabela 6 apresenta esses resultados. É possível ver que os artefatos de IHC foram usados de forma equilibrada em conjunto com as diretrizes. O elemento referente ao $\mathrm{AC}$ (Ação/X) apresentou o maior número de diferentes artefatos de IHC utilizados, o que mostra que o conjunto formado por diretrizes e artefatos de IHC foram importantes para a escrita dos AC. A técnica de Persona (P) aparece sendo aplicada em conjunto com diretrizes de todas as dimensões e em todos os elementos da UsaUS. Isto demonstra o potencial e flexibilidade desta técnica.

Para responder a (P2) (Quão fácil e útil os desenvolvedores acharam utilizar diretrizes de usabilidade para escrever as UsaUSe?) foi realizada a análise da facilidade de uso e utilidade das diretrizes a partir das respostas obtidas através do questionário baseado no modelo TAM. A utilidade percebida abrange o nível em que um indivíduo acredita que o uso de uma tecnologia específica pode melhorar seu desempenho. A facilidade de uso capta a percepção que o participante tem sobre o uso da tecnologia sem demandar esforços [Davis 1989]. A Figura 3 apresenta as respostas dadas pelos participantes em números absolutos por questão.

Algumas exceções podem ser vistas nas dimensões de facilidade de uso (F) e utilidade (U). Ambas as questões, F1 e F6, obtiveram 1 resultado "Discordo Amplamente" cada uma. Essas eram relacionadas a facilidade de elaborar as US e de flexibilizar seu uso. Ainda, 7 participantes apontaram o "Discordo Parcialmente" para a F2 sobre conseguir usar as diretrizes como queria. Analisando o perfil dos participantes que apontaram 
VIII Congresso Brasileiro de Informática na Educação (CBIE 2019)

Anais do XXX Simpósio Brasileiro de Informática na Educação (SBIE 2019)

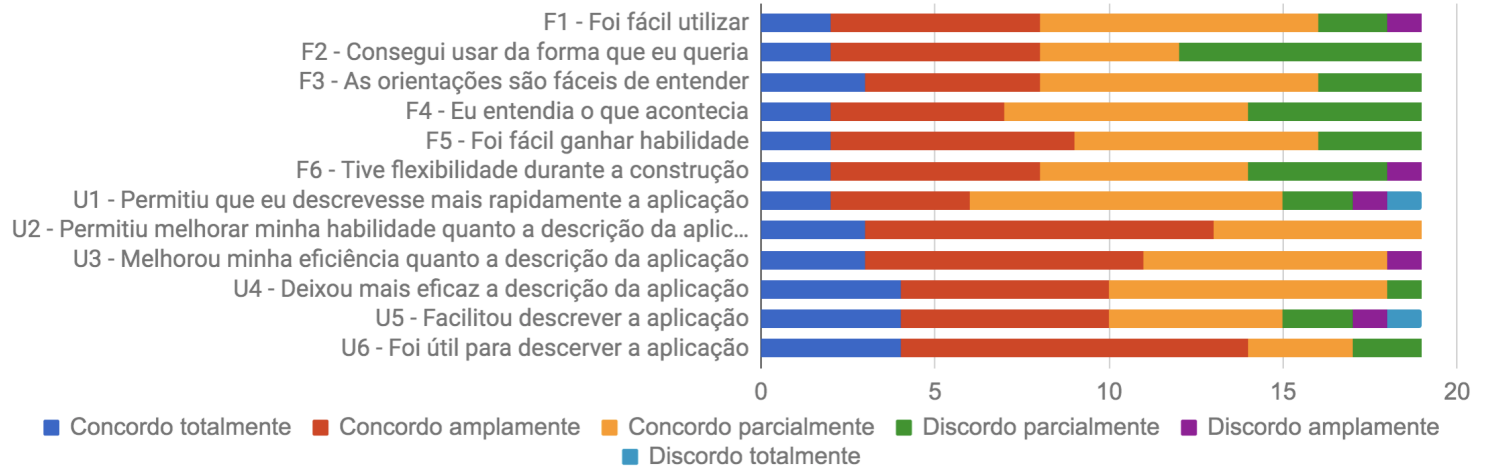

Figura 3. Facilidade de uso e utilidade da proposta percebida pelos participantes

discordância nessas questões, foi possível notar que estes tinham conhecimento teórico sobre escrita de US, mas não de técnicas/métodos de IHC. Para a dimensão de utilidade, observa-se que para a questão U1, 1 participante selecionou a opção "Discordo Totalmente" , 1 a "Discordo Amplamente" e 2 a "Discordam Parcialmente". Ou seja, para os participantes o uso das diretrizes não tornou a tarefa de escrita mais rápida. Para a questão U5, 1 participante escolheu a opção "Discordam Parcialmente"e 1 a "Discordo Amplamente", o que demonstra que ambos não consideraram que as diretrizes facilitaram a sua tarefa. Considerando o perfil destes participantes, constatou-se que 2 deles eram os mesmos que haviam apontado discordância na dimensão de facilidade de uso. Para os outros 3, observou-se que tinham conhecimento prático sobre o uso das técnicas de IHC.

A partir das discussões anteriores considera-se que a proposta UsaUSe como apoio ao desenvolvimento para aplicações de e-learning pode trazer benefícios tanto para desenvolvedores quanto para usuários. Isto é fundamental para aplicações de e-learning, pois evita que uma aplicação tenha muitos problemas de usabilidade o que faz com que seu propósito de fato seja desviado. O uso da UsaUSe ajuda os desenvolvedores a preocuparem-se com a usabilidade. Além disso, essa proposta permite trabalhar com desafios de especificidade do domínio de e-learning que conta com diferentes stakeholders envolvidos e suas diferentes visões para o mesmo software e a lacuna existente em pesquisas científicas e aplicadas sobre a adoção das técnicas e métodos de ES para o desenvolvimento de aplicações e-learning [Dalmon et al. 2012]. A gramática da US contribui para que a funcionalidade seja pensada coletando as informações relevantes da aplicação de e-learning. A US proposta possibilita uma articulação entre artefatos de IHC e diretrizes de usabilidade. A US funciona como um artefato container que concentra tal articulação. As diretrizes junto com os artefatos de IHC contribuem para que os desenvolvedores preocupem-se com: a parte visual da aplicação (Dimensão Apresentação e artefato de 10 heurísticas de Nielsen), a navegação e conteúdo (Dimensão Hipermídia e Card-Sorting), as atividades dispostas para diferentes perfis de aprendizagem (Dimensão Proatividade da aplicação e Persona) e as necessidades dos estudantes e professores voltadas para suporte ao ensino-aprendizagem (Dimensão Atividade do usuário e Questionário).

\section{Conclusões e Trabalhos Futuros}

O artigo apresentou uma proposta para escrita de US que contemplasse a descrição de requisitos de usabilidade dentro do domínio de desenvolvimento de software para $e$ - 
VIII Congresso Brasileiro de Informática na Educação (CBIE 2019)

Anais do XXX Simpósio Brasileiro de Informática na Educação (SBIE 2019)

learning. Notou-se que, como as diretrizes de usabilidade eram focadas no domínio da aplicação, estas possibilitaram que os participantes tivessem insights pertinentes ao as necessidades do domínio específico. A usabilidade deve ser considerada transversal ao desenvolvimento de software e está diretamente relacionado ao domínio. A proposta apresentada neste artigo adapta um artefato considerado importante dentro do DAS para ser usado dentro do domínio de software para e-learning. Isto faz com que a aceitação do uso da proposta seja potencializada. Como trabalhos futuros, pretende-se realizar uma análise aprofundada nas USs geradas para verificar quais requisitos de usabilidade foram descritos.

\section{Agradecimentos}

O autores agradecem o suporte financeiro da Coordenação de Aperfeiçoamento de Pessoal de Nível Superior - Brasil (CAPES) - Código de Financiamento 001. E também o apoio financeiro através dos processos no 2013/25572-7, n 2017/03397-0 e n 2014/25779-3, Fundação de Amparo à Pesquisa do Estado de São Paulo (FAPESP).

\section{Referências}

9241-210, I. (2009). 9241-210: 2010. ergonomics of human system interaction-part 210: Human-centred design for interactive systems. International Standardization Organization (ISO). Switzerland.

Ardito, C., Costabile, M. F., De Marsico, M., Lanzilotti, R., Levialdi, S., Roselli, T., and Rossano, V. (2006). An approach to usability evaluation of e-learning applications. Universal access in the information society, 4(3):270-283.

Brito, M. C. A. (2013). Development of a methodology for software development to factory software an educational institution. Brazilian Journal of Computers in Education, 21(2):52-61.

Chimalakonda, S. and Nori, K. V. (2012). A software engineering perspective for accelerating educational technologies. In 2012 IEEE 12th International Conference on Advanced Learning Technologies, pages 754-755. IEEE.

Choma, J., Zaina, L. A., and Beraldo, D. (2016). Userx story: incorporating ux aspects into user stories elaboration. In International Conference on Human-Computer Interaction, pages 131-140. Springer.

Cohn, M. (2009). User stories applied: For agile software development.

Dalmon, D. L., Brandão, A. A., and Brandão, L. O. (2012). Uso de métodos e técnicas para desenvolvimento de software educacional em universidades brasileiras. In Anais do Workshop de Desafios da Computação Aplicada à Educação, pages 236-245.

Dalmon, D. L. and Brandão, L. d. O. (2013). On the development of educational software: proposal of a software product line for interactive learning modules. Brazilian Journal of Computers in Education, 21(3):113-130.

Davis, F. D. (1989). Perceived usefulness, perceived ease of use, and user acceptance of information technology. MIS quarterly, pages 319-340.

Dodero, J., Garcia-Penalvo, F., Gonzalez, C., Moreno-Ger, P., Redondo, M., SarasaCabezuelo, A., and Sierra, J. (2014). Development of e-learning solutions: Different 
VIII Congresso Brasileiro de Informática na Educação (CBIE 2019)

Anais do XXX Simpósio Brasileiro de Informática na Educação (SBIE 2019)

approaches, a common mission, tecnologias del aprendizaje. IEEE Revista Iberoamericana de, 9(2).

Gonçalves, F. M., Arpetti, A., and Baranauskas, M. C. C. (2014). Facilitando a construção social de significado em sistemas de aprendizado colaborativo. In Proceedings of the xix international workshop on educational software, tise, volume 10, pages 318-326.

Gordillo, A., Barra, E., Aguirre, S., and Quemada, J. (2014). The usefulness of usability and user experience evaluation methods on an e-learning platform development from a developer's perspective: A case study. In 2014 IEEE Frontiers in Education Conference (FIE) Proceedings, pages 1-8. IEEE.

Hudson, W. (2013). User stories don't help users: introducing persona stories. interactions, 20(6):50-53.

Moreno, A. M. and Yagüe, A. (2012). Agile user stories enriched with usability. In International Conference on Agile Software Development, pages 168-176. Springer.

Nakamura, W. T., de Oliveira, E. H. T., and Conte, T. (2017). Usability and user experience evaluation of learning management systems. In Proc. of the 19th International Conference on Enterprise Information Systems, volume 3, pages 97-108.

Quiñones, D. and Rusu, C. (2017). How to develop usability heuristics: A systematic literature review. Computer Standards \& Interfaces, 53:89-122.

Ribeiro, S. and Melo, A. (2017). Um método para o desenvolvimento de software com crianças utilizando o ambiente scratch. In Brazilian Symposium on Computers in Education (Simpósio Brasileiro de Informática na Educação-SBIE), volume 28, page 1027.

Rogers, Y., Sharp, H., and Preece, J. (2013). Design de Interação. Bookman Editora.

Santos, F., Rosa, J., Silva, G., and Matos, E. (2017). Aplicando o spide à engenharia de requisitos de um ambiente virtual de aprendizagem: resultados preliminares. In Anais dos Workshops do Congresso Brasileiro de Informática na Educação, volume 6, page 1364.

Sarasa-Cabezuelo, A. and Sierra-Rodríguez, J. L. (2013). Software engineering for elearning. In Proceedings of the First International Conference on Technological Ecosystem for Enhancing Multiculturality, pages 81-86. ACM.

Schön, E.-M., Thomaschewski, J., and Escalona, M. J. (2017). Agile requirements engineering: A systematic literature review. Computer Standards \& Interfaces, 49:79-91.

Silva, J., Rangel, L. L., and Marques, A. B. (2018). Utilizando design science na criação de um artefato para elicitação de requisitos para jogos educacionais digitais. In Anais do XXIX Simpósio Brasileiro de Informática na Educação (SBIE 2018), pages 476485.

Van Nuland, S. E., Eagleson, R., and Rogers, K. A. (2017). Educational software usability: Artifact or design? Anatomical sciences education, 10(2):190-199.

Zaina, L., Leles, A., Duarte, A., Góis, G., and Welter, E. (2015). Usability and user experience evaluation of learning management systems. In Anais do XXVI Simpósio Brasileiro de Informática na Educação (SBIE 2015), pages 51-60. 